\title{
Spiny bottlenecks for calcium
}

Dendritic spines move; stubby, thin or mushroom alike. The question is what is the purpose of their relentless motility. Some clues have come from the observation that spines move during synaptogenesis and in response to synaptic stimulation. However, it is not clear whether this motility has any effect in the functional properties of the spine and the dendrite. To approach this problem, Majewska et al. have analysed the way in which diffusion into and out of the spine is altered in response to changes in the length of the spine neck using two independent strategies.

First, the authors transfected cortical cultured slices with enhanced green fluorescent protein, photobleached the fluorescence from individual spines, and measured the time constant for the recovery of the label. They observed a positive correlation between the time constant and the length of the spine. Furthermore, they studied the same population of spines at different time points to find out whether the continuous changes in length would have a similar dynamic effect on diffusion. Again, they found that a given change in neck length was accompanied by a proportional change in the time constant for diffusion.

Second, Majewska et al. evoked calcium transients in spines of neurons from acute slices by electrical stimulation. They found that the fast component of calcium decay showed a clear correlation to the length of the spine and that motility also had a dynamic effect on diffusion.

As one of the main functions of spines may be to act as biochemical compartments, the effect of length on calcium dynamics could have profound implications for synaptic function and plasticity. At the same time, the fact that bidirectional changes in length occur within minutes indicates that the efficacy of a given synapse might be subject to a more dynamic regulation than commonly thought. Moreover, their recent observation that Rho GTPases are involved in the regulation of spine length will enable us to determine how spine dynamics interact with synaptic activity.

Juan Carlos López

(1) References and links

ORIGINAL RESEARCH PAPERS Majewska, A. et al. Regulation of spine calcium dynamics by rapid spine motility. J. Neurosci. 1, 1-10 (2000) | Tashiro, A. et al. Regulation of dendritic spine morphology by the Rho family of small GTPases, antagonistic roles of Rac and Rho. Cerebral Cortex 10, 927-938 (2000) | Parnass, Z et al. Analysis of spine morphological plasticity in developing hippocampal pyramidal neurons. Hippocampus 10, 561-568 (2000) FURTHER READING Svoboda, K. et al. Direct measurement of coupling between dendritic spines and shafts. Science 272, 716-719 (1996) | Yuste, R. et al. From form to function: calcium compartamentalization in dendritic spines. Nature Neurosci. 3, 653-659 (2000) ENCYCLOPEDIA OF LIFE SCIENCES Dendritic spines | Dendrites

WEB SITE Rafael Yuste's laboratory
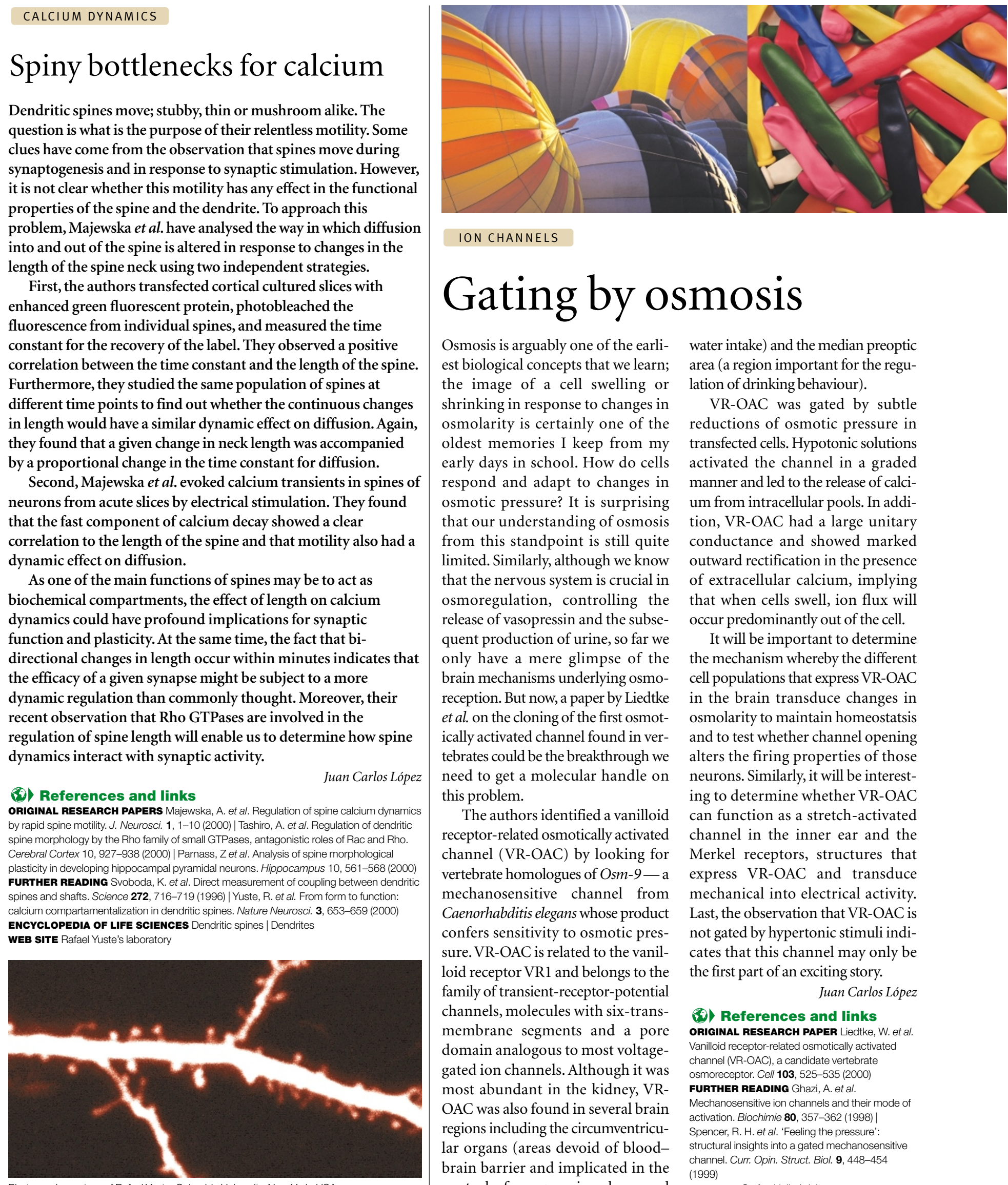

ION CHANNELS

\section{Gating by osmosis}

Osmosis is arguably one of the earliest biological concepts that we learn; the image of a cell swelling or shrinking in response to changes in osmolarity is certainly one of the oldest memories I keep from my early days in school. How do cells respond and adapt to changes in osmotic pressure? It is surprising that our understanding of osmosis from this standpoint is still quite limited. Similarly, although we know that the nervous system is crucial in osmoregulation, controlling the release of vasopressin and the subsequent production of urine, so far we only have a mere glimpse of the brain mechanisms underlying osmoreception. But now, a paper by Liedtke et al. on the cloning of the first osmotically activated channel found in vertebrates could be the breakthrough we need to get a molecular handle on this problem.

The authors identified a vanilloid receptor-related osmotically activated channel (VR-OAC) by looking for vertebrate homologues of Osm-9-a mechanosensitive channel from Caenorhabditis elegans whose product confers sensitivity to osmotic pressure. VR-OAC is related to the vanilloid receptor VR1 and belongs to the family of transient-receptor-potential channels, molecules with six-transmembrane segments and a pore domain analogous to most voltagegated ion channels. Although it was most abundant in the kidney, VROAC was also found in several brain regions including the circumventricular organs (areas devoid of bloodbrain barrier and implicated in the control of vasopressin release and water intake) and the median preoptic area (a region important for the regulation of drinking behaviour).

VR-OAC was gated by subtle reductions of osmotic pressure in transfected cells. Hypotonic solutions activated the channel in a graded manner and led to the release of calcium from intracellular pools. In addition, VR-OAC had a large unitary conductance and showed marked outward rectification in the presence of extracellular calcium, implying that when cells swell, ion flux will occur predominantly out of the cell.

It will be important to determine the mechanism whereby the different cell populations that express VR-OAC in the brain transduce changes in osmolarity to maintain homeostatsis and to test whether channel opening alters the firing properties of those neurons. Similarly, it will be interesting to determine whether VR-OAC can function as a stretch-activated channel in the inner ear and the Merkel receptors, structures that express VR-OAC and transduce mechanical into electrical activity. Last, the observation that VR-OAC is not gated by hypertonic stimuli indicates that this channel may only be the first part of an exciting story. Juan Carlos López

(9) References and links ORIGINAL RESEARCH PAPER Liedtke, W. et al. Vanilloid receptor-related osmotically activated channel (VR-OAC), a candidate vertebrate osmoreceptor. Cell 103, 525-535 (2000) FURTHER READING Ghazi, A. et al. Mechanosensitive ion channels and their mode of activation. Biochimie 80, 357-362 (1998) | Spencer, R. H. et al. 'Feeling the pressure': structural insights into a gated mechanosensitive channel. Curr. Opin. Struct. Biol. 9, 448-454 (1999) WEB SITE Stefan Heller's laboratory 